Research Article

\title{
Steroid nasal spray versus curettage adenoidectomy in school children - a randomized controlled trial
}

\begin{abstract}
Background: Management of chronic adenoiditis is planned according to the degree of airway obstruction, age of the patient and related morbidity. Considering the individual risk analysis of patients in terms of anesthetic, post-operative complications and recurrence, non-surgical treatment could be considered in patients who are unfit for surgery.

Methods: Randomized control trial was conducted to compare the effects of topical nasal steroid spray with curettage adenoidectomy in chronic adenoiditis. Post intervention, patients were followed up for 6months.

Results: Out of 103 patients, 51 were subjected to adenoidectomy and 52 to medical treatment. At the end of 6months, 49 (Negative rank score) patients of surgical group and 48 (Negative rank score) patients of medical group showed statistically significant improvement $(\mathrm{p}<.001)$ in symptoms and regression of adenoid size. There was no statistically significant difference between groups with respect to recurrent cold, running nose, epistaxis and ear symptoms $(\mathrm{p}>.05)$.

Conclusion: Topical nasal steroid spray can be used as an alternative treatment in chronic adenoiditis when surgery is contraindicated. It can also be considered when surgery needs to be postponed at the request of the patient.
\end{abstract}

Keywords: adenoidectomy, nasal steroid spray, nasopharynx
Volume 8 Issue 5 - 2017

\section{Vikram Kemmannu Bhat, Arshad Muhammed Razi,Vibhor Basant Kumar}

Department of Otolaryngology Karnataka Institute of Medical

Sciences, India

Correspondence: Vikram Kemmannu Bhat Department of Otolaryngology Karnataka Institute of Medical Sciences Vidyanagar Hubli 58002I, India, Email vikram.ent@gmail.com

Received: August 20, 2017| Published: September 18, 2017

\section{Introduction}

The nasopharyngeal tonsil, or adenoid, is a lympho epithelial organ situated in a critical anatomical position i.e., the roof and posterior wall of nasopharynx. The adenoid is closely related to nasopharyngeal orifice of the Eustachian tube and paranasal sinuses ostia, particularly the ethmoid and sphenoid sinuses. The adenoid being a component of Waldeyer ring possesses particular immunological properties and plays an important role not only in local, but also in general immune system.

Recurrence of adenoiditis is more common if children get operated at a young age. ${ }^{1}$ Recent studies have also shown hypertrophy of other lymphoid tissues in the Waldeyer ring following adenoidectomy. ${ }^{2}$ In the developing countries, there are a number of young children presenting with mouth breathing and running nose, especially in the rural and urban slum areas where the socioeconomic conditions and the healthcare facilities are poor. Almost all of them have been found to have chronic adenoiditis and some of them also have associated chronic sinusitis, otitis media with effusion and obstructive sleep apnea.

The aim of the study was to find out if intranasal topical steroid spray could be used to postpone or to avoid unnecessary adenoidectomy, especially in young children where surgery is not desirable or contraindicated.

In various studies the use of topical application of intranasal steroid spray in chronic adenoiditis has been proved. ${ }^{3}$ However, whether it can also be used to postpone surgery due to some contraindication has not been studied. The study was designed to compare the outcomes of topical intranasal steroid spray and adenoidectomy in chronic adenoiditis.

\section{Materials and methods}

Cases of chronic non-recurrent adenoiditis attending Ear, Nose $\&$ Throat outpatient clinic of tertiary care institute were recruited into the study. This was a randomized controlled trial spanning one year. Ethical clearance for the study was obtained from the local institutional review board.

Prospective study candidates were examined and investigated in the ENT outpatient clinic to confirm the eligibility. Diagnostic nasal endoscopy (DNE) was performed in all of them. Adenoid hypertrophy was graded into four grades according to the Parikh's classification system $^{4}$ (Table $1 \&$ Figure 1).

Table I Parikh's classification

\begin{tabular}{ll}
\hline Grade & $\begin{array}{l}\text { Description of Anatomic Structures } \\
\text { in Relation to Adenoids }\end{array}$ \\
\hline 1 & $\begin{array}{l}\text { Adenoids are non-obstructive and not in contact with } \\
\text { Torus Tubarius }\end{array}$ \\
\hline 2 & Adenoids in contact with Torus Tubarius \\
\hline 3 & Adenoids in contact with Torus Tubarius and Vomer \\
\hline 4 & $\begin{array}{l}\text { Adenoids in contact with Torus Tubarius, } \\
\text { Vomer and Soft Palate }\end{array}$ \\
\hline
\end{tabular}

\section{Inclusion criteria}

i. Adenoid enlargement grade $2 \& 3$.

ii. Age between 6-15yrs.

iii. Patients who were eligible and willing for either treatment groups

iv. Immuno-competent and non-recurrent cases. 


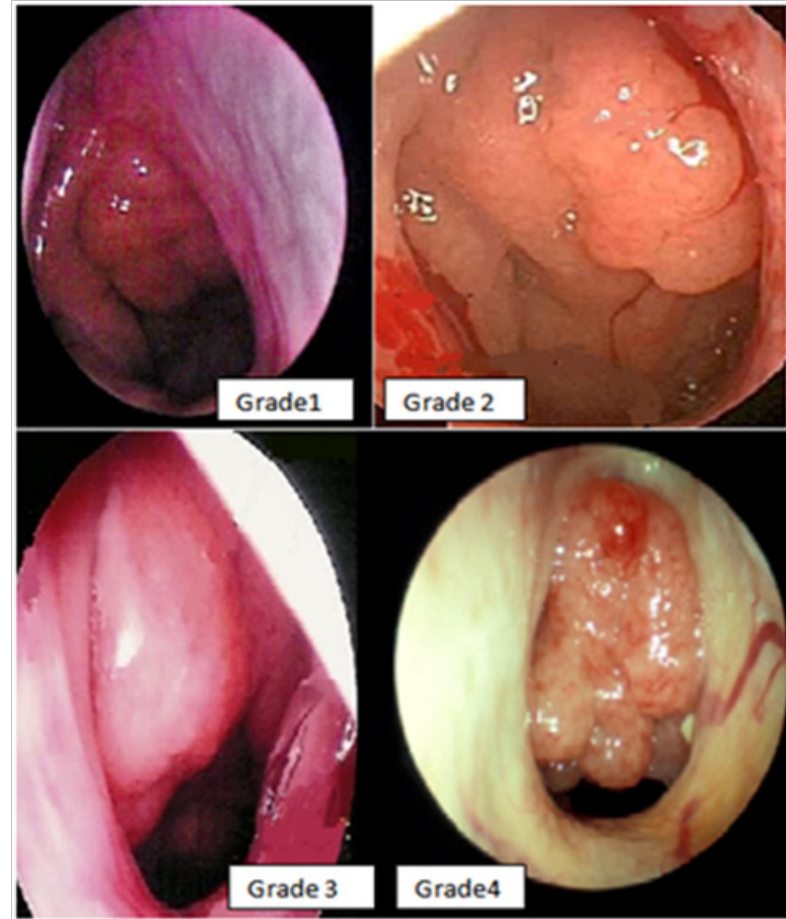

Figure I Different grades of adenoids hypertrophy.

\section{Exclusion criteria}

A. Acute adenoiditis

B. Enlargement of components of Waldeyer ring other than adenoids

C. Adenoid obstructing choana completely (Grade IV) and minimally enlarged adenoid (Grade I).

A written consent was taken from the parents. During flexible nasopharyngoscopy the following observations were made and recorded.

a. Adenoid hypertrophy grade

b. Eustachian tube function on swallowing

c. Posterior pharyngeal wall (Nodules / post nasal drip)

d. Posterior cushion thickening \& obliteration of Fossa of Rosenmuller.

e. Tubal tonsil status.

All patients underwent audiological evaluation by pure tone audiometry \& immittance. Hundred and three patients were randomized to one of the two treatment groups. Group one (Medical treatment - steroid spray) and group two (Surgical treatmentadenoidectomy). A random sequence of the numbers one and two was generated using RALLOCsoftware. Number one corresponded to medical treatment (Group 1) and number two to surgical treatment (Group 2). Patients underwent one of the two treatment options as per their computer generated, random allocation number.

All computer generated random numbers were carefully marked in a paper slip. This paper slip was then sealed in an opaque, dark envelope. The numbered envelopes were tagged together in the ascending order. These steps were performed by a neutral observer who was not involved in the study.
Patients selected for medical treatment for chronic adenoiditis were prescribed Fluticasone Furoate nasal steroid spray. A detailed instruction was given to the patients and their attendants about the usage of the spray, need for continuous usage for specified period, need for regular follow up and exact dates of follow up. Patients were advised to use $100 \mathrm{mcg}$ of Fluticasone Furoate once daily as one spray in each nostril morning and evening for 12 weeks. Standard rescue medications were permitted in case of distress in all patients. Medical treatment failures and relapses were advised to undergo surgery after 6months.

Patients selected for surgical treatment were subjected to curettage adenoidectomy under general anesthesia by a single experienced surgeon. The same surgical protocol and postoperative management was followed in all surgeries. Intraoperatively, complete clearance of adenoid was ensured by digital palpation and endoscopy after surgery. Patient was observed for 24 hours and then discharged.

Patients were followed up in the ENT outpatient clinic at one, three and six months post intervention. Every visit was well documented and DNE video recorded for future analysis. Intention to treat analysis method was adopted (Last response carry forward) to accommodate the drop outs.

\section{Primary outcomes}

1. Adenoid size and presence as assessed by DNE.

2. Improvement of signs and symptoms (Mouth breathing, snoring, nasal obstruction - Visual analogue score (VAS), recurrent cold, epistaxis, hyponasal speech)

3. Recurrent \& residual disease after treatment assessed by DNE.

\section{Secondary outcomes}

1. Hearing Improvement-Pure tone audiogram (PTA)

2. Earache-VAS

3. Complications of treatment in any of the groups

4. Requirement for rescue medication

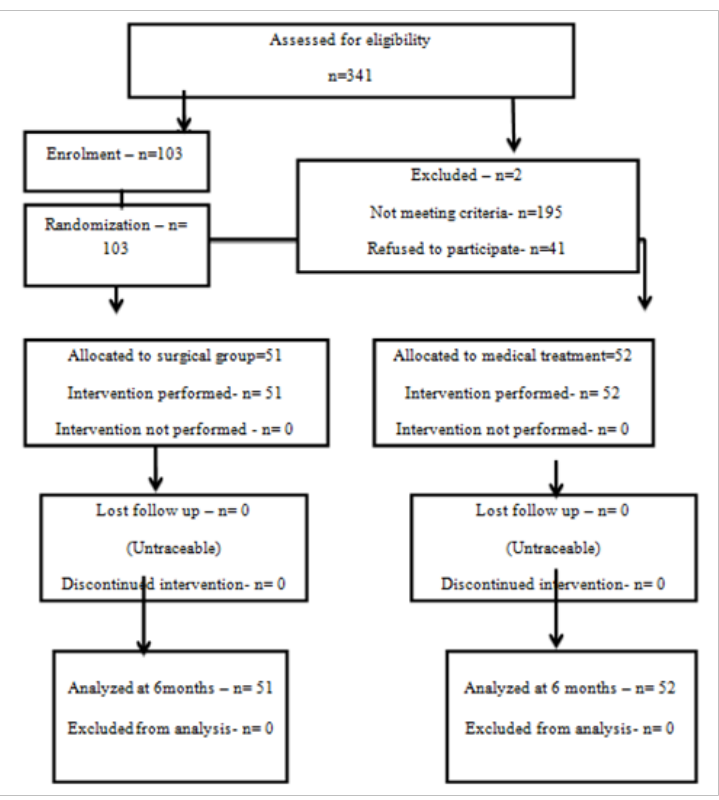

Figure 2 Consort flow chart showing study details. 
The study was completed and recorded in accordance with the revised CONSORT statement guidelines (Figure 2) for evaluating the structure and analysis of randomized controlled trials. SPSS version 20.0 was used for statistical analysis.

\section{Results}

In our study the minimum age was 6years and maximum age was 15years. The maximum incidence was at 11-12years of age, 54.4 $\%$ of those included in our study were males whereas $45.65 \%$ were females. Mean age in surgical group was $10.6 \pm 2.49$ and medical treatment group was $11.40 \pm 2.6$.

Final analysis of the study was done by taking all the symptoms into consideration, the total symptoms score of each patient before and after treatment was calculated and compared within the group and between the groups. Scoring for eight symptoms (Mouth breathing, snoring, nasal obstruction, earache, hearing improvement, running nose, rhinolalia clausa and epistaxis) was considered (Table 2). Scoring system comprised of four scores according to severity of symptoms. Score 0 implied no symptom and score 3 with maximum severity.
For analyzing the consolidated symptoms score (primary outcomes), within each group separately, Wilcoxon Signed Ranked Test was used. In surgical group, 49 out of 51 showed significant improvement in symptom scores (Table 3 ) after the follow up period. The difference between pre and post intervention symptom score was statistically significant ( $\mathrm{p}$ value $<.000$ ). Two patients in surgical group presented with recurrence of disease during follow up of six months.

In medical group, 48 of total 52 patients showed improvement in symptoms score (Table 4) during the treatment period. Four of them did not show any improvement of symptoms during treatment. There was statistically significant difference between pre and post intervention symptom score ( $\mathrm{p}$ value $<.000$ ). In this group, 15 patients had a relapse after the cessation of nasal steroid spray.

For overall comparison between the outcomes of medical and surgical group, Mann Whitney U test was used (Table 5). The reduction in median score in surgical group was eight and medical group was six. There was significant reduction in total score of both surgical and medical groups but, surgical treatment group showed higher improvement than medical treatment during 24 weeks follow up period.

Table 2 Comparison of outcomes between groups

\begin{tabular}{|c|c|c|c|}
\hline \multicolumn{4}{|l|}{ Primary Outcomes } \\
\hline \multirow[t]{2}{*}{ Outcome } & \multirow[t]{2}{*}{$P$ value } & \multicolumn{2}{|c|}{ Percentage of Improvement in Symptoms between Groups } \\
\hline & & Surgical & Medical \\
\hline Mouth breathing & 0.001 & $96 \%$ & $71 \%$ \\
\hline Snoring & 0.001 & $98 \%$ & $66 \%$ \\
\hline Nasal obstruction & 0.001 & $96 \%$ & $65 \%$ \\
\hline Recurrent cold & 0.086 & $93 \%$ & $85 \%$ \\
\hline Epistaxis & 0.999 & $100 \%$ & $89 \%$ \\
\hline Rhinolalia clausa & 0.015 & $94 \%$ & $67 \%$ \\
\hline Adenoid size regression & 0.001 & $96 \%$ & $71 \%$ \\
\hline Running nose & 0.03 & $95 \%$ & $71 \%$ \\
\hline \multicolumn{4}{|l|}{ Secondary Outcomes } \\
\hline Hearing improvement & 0.999 & $100 \%$ & $89 \%$ \\
\hline Posterior pharyngeal wall nodule regression & 0.39 & $59 \%$ & $57 \%$ \\
\hline
\end{tabular}

Table 3 Comparison of consolidated clinical features before and after treatment within surgical group

\begin{tabular}{lllllll}
\hline & & N & Mean Rank & Sum of Ranks & Z & $\begin{array}{l}\text { Asym.Sig } \\
\text { (Two-Tailed) }\end{array}$ \\
\hline Post-Pre & Negative Ranks & 49a & 25.98 & 1273 & $-6.147 \mathrm{~b}$ & 0 \\
\hline & Positive Ranks & Ib & 2 & 2 & & \\
\hline Ties & Ic & & & & \\
\hline Total & 5I & & & & \\
\hline
\end{tabular}

Table 4 Comparison of consolidated clinical features before and after treatment within medical group

\begin{tabular}{lllllll}
\hline & N & Mean Rank & Sum of Ranks & z & Asym.sig \\
\hline \multirow{2}{*}{ Post-pre } & & & & & (Two-Tailed) \\
& Negative Ranks & $48 \mathrm{a}$ & 25.35 & 1217 & $-6.147 \mathrm{~b}$ & 0 \\
& Positive Ranks & Ib & 8 & 8 & $147 \mathrm{~b}$ & 0 \\
& Ties & $3 \mathrm{c}$ & & & & \\
& Total & 52 & & & & \\
\hline
\end{tabular}


Table 5 Comparison of consolidated clinical features between the groups

\begin{tabular}{|c|c|c|c|c|c|c|c|c|}
\hline & & $\mathbf{N}$ & Mean & SD & 25 & 50 (Median) & 75 & Mann whitey $U$ test \\
\hline \multirow[t]{2}{*}{0} & Medical & 52 & 8.33 & 2.15 & 7 & 8 & 10 & $P=0.64$ \\
\hline & Surgical & 51 & 8.53 & 2.21 & 7 & 8 & 10 & \\
\hline \multirow[t]{2}{*}{6 Months } & Medical & 52 & 2.63 & 3.74 & 0 & 0 & 6.75 & $P=0.001$ \\
\hline & Surgical & 51 & 0.29 & 1.38 & 0 & 0 & 0 & \\
\hline Reduction in Score & Medical & 52 & 5.69 & 3.94 & 2.25 & 6 & 9 & $P=0.00 I$ \\
\hline$(0-6)$ & Surgical & $5 I$ & 8.23 & 2.7 & 7 & 8 & 10 & \\
\hline
\end{tabular}

\section{Discussion}

Chronic adenoiditis and its associated complications are one of the most common entities encountered by an otorhinolaryngologist in day to day practice. Adenoidectomy is conventionally believed to be the standard treatment for chronic adenoiditis and its secondary effects. Curettage adenoidectomy even though imperfect, continues to be popular especially in the developing world. It is cost effective and faster as compared to the newer techniques. This method was adopted in this study as it was the standard protocol in the institute.

A recent study conducted in the institute had shown hypertrophy of other components of Waldeyer ring after adenoidectomy or adenotonsillectomy. ${ }^{5}$ Hypertrophy of tubal tonsil was one of the important observations in the study. The physiological reasons for the hypertrophy and clinical significance need to be studied further It would be prudent to delay adenoidectomy in young children with minimal to moderate disease and give medical treatment a chance. Currently there is no consensus and no proper treatment guidelines that are universally accepted for chronic adenoiditis in literature. Adenoidectomy is accepted as the standard of care in conditions like obstructive sleep apnea syndrome and cardio-respiratory complications associated with severe chronic adenoid hypertrophy. ${ }^{6}$ However, in less severe cases of chronic adenoiditis, non-surgical treatment could be tried. The effect of nasal steroid spray for chronic adenoiditis and allergic rhinitis has been proved by various randomized control trials. ${ }^{7}$ One of the challenges faced in this study, was the counselling of patient to accept either of the 2 treatment groups allocated by randomization.

Most of the studies reported in the past compared a topical nasal steroid spray with a placebo like saline drops. These studies obviously reported in favor of topical steroid spray. Six randomized control trials $^{7}$ involving a total of 394 patients were available in literature; five of the six trials ${ }^{8-13}$ demonstrated a significant proof of intranasal corticosteroid in improving nasal obstruction symptoms and in reducing adenoid size. To the best of our knowledge this is the first study to report comparison of medical and surgical treatment.

Even though Table 5 depicts the superiority of surgical treatment over medical treatment, it also shows that the latter could achieve significant $(75 \%)$ reduction in mean symptom score. This proves that it can achieve remission for considerable periods and could also help tide over acute episodes.

The long-term efficacy of intranasal corticosteroids is an issue that needs further studies. Marco Berlucchi et al. ${ }^{14}$ reported the first longterm follow-up of pediatric patients undergoing steroid nasal spray treatment for adenoid problems. Proper treatment under close medical supervision may obtain successful results. Such long-term therapy is safe and well tolerated by pediatric patients. ${ }^{14}$

The use of Fluticasone Furoate spray has been previously reported in literature. It has been shown to be well tolerated in children, adolescents and adults with an overall incidence of adverse events similar to that of placebo. ${ }^{15}$ Most common adverse effects were headache, epistaxis, nasopharyngitis, pyrexia, pharyngolaryngeal pain, nasal ulceration and cough.

\section{Conclusion}

Topical nasal steroid spray for grade 2 and 3 chronic adenoiditis is a safer, simpler and painless alternative to surgery to achieve remissions in young children. This is more relevant in the developing countries where the burden of disease in the younger age is high and frequently a cause of running nose and snoring. It can also be useful where surgery is contraindicated in conditions like bleeding disorders, cleft palate and bifid uvula. Conservative treatment has the advantage of not having the complications of surgery like bleeding and hypertrophy of other components of Waldeyer ring. It is useful to control concurrent conditions of the nose and PNS like allergic rhinitis and sinusitis, which have a significant contribution towards a running nose especially in children. A conservative attitude towards chronic adenoiditis appears immunologically desirable, especially at a young age.

\section{Conflicts of interest}

None to declare.

\section{Acknowledgments}

None.

\section{Funding}

None.

\section{References}

1. Duval M, Chung JC, Vaccani JP. A case-control study of repeated adenoidectomy in children. JAMA Otolaryngol Head Neck Surg. 2013;139(1):32-36.

2. Emerick KS, Cunningham MJ. Tubal Tonsil Hypertrophy-A Cause of Recurrent Symptoms after Adenoidectomy. Arch Otolaryngol Head Neck Surg. 2006;132(2):153-156.

3. Burton MJ, Derkay CS, Rosenfeld RM. Intranasal corticosteroid spray for moderate to severe adenoidal hypertrophy. Otolaryngol Head Neck Surg. 2009;140:451.

4. Parikh SR, Coronel M, Lee JJ, et al. Validation of a new grading system for endoscopic examination of adenoid hypertrophy. Otolaryngol Head Neck Surg. 2006;135(5):684-687.

5. Bhat KV, Shetty D, Nagaiah PH. Effects of adenotonsillectomy on the components of Waldeyer ring. Int J Otorhinolaryngol Head Neck Surg. 2017;3(2):290-297.

6. Gerhardsson H, Stalfors J, Odhagen E, et al. Pediatric adenoid surgery in Sweden 2004-2013: Incidence, indications and concomitant surgical procedures. Int J Pediatr Otorhinolaryngol. 2016;87:61-66. 
7. Zhang L, Mendoza-Sassi RA, Cesar JA, et al. Intranasal corticosteroids for nasal airway obstruction in children with moderate to severe adenoidal hypertrophy. Cochrane Database Syst Rev. 2008;16(3):CD006286.

8. Demain JG, Goetz DW. Pediatric adenoidal hypertrophy and nasal airway obstruction: reduction with aqueous nasal beclomethasone. Pediatrics. 1995;95(3):355-364.

9. Demirhan H, Aksoy F, Özturan O, et al. Medical treatment of adenoid hypertrophy with fluticasone propionate nasal drops. Int $J$ Pediatr Otorhinolaryngol . 2010;74(7):773-776.

10. Berlucchi M, Salsi D, Valetti L, et al. The role of mometasonefuroate aqueous nasal spray in the treatment of adenoidal hypertrophy in the pediatric age group: preliminary results of a prospective, randomized study. Pediatrics. 2007;119(6):e1392-e1397.

11. Ciprandi G, Varricchio A, Capasso M, et al. Intranasal flunisolide treatment in children with adenoidal hypertrophy. Int J Immunopathology and Pharmacol. 2007;20(4):833-836.
12. Criscuoli G, D'Amora S, Ripa G, et al. Frequency of surgery among children who have adenotonsillar hypertrophy and improve after treatment with nasal beclomethasone. Pediatrics. 2003;111:e236-e238.

13. Lepcha A, Kurien M, Job A, et al. Chronic adenoid hypertrophy in children - is steroid nasal spray beneficial? Indian $J$ Otolaryngol Head and Neck Surg. 2002;54(4):280-284.

14. Berlucchi M, Valetti L, Parrinello G, et al. Long-term follow-up of children undergoing topical intranasal steroid therapy for adenoidal hypertrophy. Int J Pediatr Otorhinolaryngol. 2008;72(8):1171-1175.

15. Rosenblut A, Bardin PG, Muller B, et al. Long term safety of fluticasone furoate in adult and adolescents with perennial allergic rhinitis. Allergy.

16. $2007 ; 62(9): 1071-1077$. 